\title{
Strigolactone and Ethylene Inhibitor Suppressing Dark-induced Leaf Senescence in Perennial Ryegrass Involving Transcriptional Downregulation of Chlorophyll Degradation
}

\author{
Qiannan Hu \\ Department of Plant Biology, Rutgers University, 59 Dudley Road, New Brunswick, NJ 08901; and \\ College of Forestry, Northwest A\&F University, Yangling, Shaanxi 712100
}

Fei Ding

College of Forestry, Northwest A\&F University, Yangling, Shaanxi 712100, China

Mingna Li

Department of Plant Biology, Rutgers University, 59 Dudley Road, New Brunswick, NJ 08901

Xiaxiang Zhang

Department of Plant Biology, Rutgers University, 59 Dudley Road, New Brunswick, NJ 08901; and

College of Agro-grassland Science, Nanjing Agricultural University, Nanjing, Jiangsu 210095, China

\author{
Shuoxin Zhang \\ College of Forestry, Northwest A\&F University, Yangling, Shaanxi 712100, China; and Qinling \\ National Forest Ecosystem Research Station, Ningshan, Shaanxi 711600, China
}

\author{
Bingru Huang \\ Department of Plant Biology, Rutgers University, 59 Dudley Road, New Brunswick, NJ 08901
}

\begin{abstract}
ADDITIONAL INDEX wORDs. aminoethoxyvinyl glycine, gene expression, GR24, Lolium perenne
Abstract. Accelerated or premature leaf senescence induced by dark conditions could be associated with chlorophyll degradation and regulated by hormones. To study the effects of strigolactone (SL) on dark-induced leaf senescence and to examine the interaction effects of SL and ethylene on regulating dark-induced leaf senescence, plants of perennial ryegrass (Lolium perenne) exposed to darkness for 8 days were treated with a synthetic SL analogue (GR24), aminoethoxyvinyl glycine [AVG (an ethylene biosynthesis inhibitor)], or SL and AVG by foliar spray. Chlorophyll content, photochemical efficiency, electrolyte leakage, and ethylene production were measured. Expressions of genes associated with leaf senescence, SL biosynthesis and signaling, ethylene biosynthesis and signaling, and chlorophyll biosynthesis and degradation were determined. Foliar application of GR24 promoted leaf senescence in perennial ryegrass grown in darkness, and the intensity of action increased with the GR24 concentration. SL-accelerated leaf senescence was associated with the downregulation of four chlorophyll biosynthesis-associated genes and upregulation of four chlorophyll degradation-associated genes. AVG had functions counteractive to SL, suppressing darkinduced leaf senescence by downregulating chlorophyll degradation genes and SL synthesis genes. Our results suggested that SL and ethylene interactively regulated leaf senescence, mainly by controlling chlorophyll degradation induced by darkness in perennial ryegrass.
\end{abstract}

Leaf senescence is regulated by hormones and is also inducible by environmental stresses, such as low light (Wingler et al., 1998). Strigolactone (SL) was first discovered as a stimulant of seed germination in some plant species, such as witchweed [Striga lutea (Cook et al., 1966)] and broomrape

Received for publication 17 Apr. 2020. Accepted for publication 9 June 2020. Published online 6 January 2021.

This research was supported by Rutgers Center for Turfgrass Science, National Key R\&D Program of China [2016YFC0500202], and Qinling National Forest Ecosystem Research Station of China. We thank Stephanie Rossi and William Errickson at Rutgers University for critical review and editing of the manuscript.

B.H. is the corresponding author. E-mail: huang@sebs.rutgers.edu.

This is an open access article distributed under the CC BY-NC-ND license (https://creativecommons.org/licenses/by-nc-nd/4.0/).
[Orobanche ramose (Xie et al., 2010)]. Recent research found that SL is a plant hormone that has multiple roles in plant development, like promoting seed germination (Toh et al., 2012), altering plant architecture (Hu et al., 2018; Umehara et al., 2008), and enhancing plant stress tolerance to drought, salt, heat, phosphate deficiency, and nitrate deficiency (Ha et al., 2014; Hu et al., 2018; Sun et al., 2014). Several studies reported that SL may also be involved in regulating leaf senescence. Mutant plants deficient in SL biosynthesis or insensitive to SL signaling like $\max 2$ and $d 3$ showed delayed leaf senescence compared with the wild type in several plant species (Liu et al., 2013; Snowden et al., 2005, 2012; Woo et al., 2001; Yan et al., 2007). The application of a synthetic SL analogue (GR24) promoted leaf senescence in SL-deficient mutants like $d 27, d 17$, and $d 10$ in rice (Oryza sativa) and maxl, 
max3, and max4 in arabidopsis (Arabidopsis thaliana), but not in SL-signaling mutants like $d 3$ and $d 14$ in rice or max 2 and atd14 in arabidopsis (Ueda and Kusaba, 2015; Yamada et al., 2014). However, there is no report of the altered senescence phenotype for SL mutants or transgenic plants in pea (Pisum sativum) or tomato (Solanum lycopersicum) (Beveridge et al., 1997; Kohlen et al., 2012; Vogel et al., 2010). Several senescence-associated genes, such as $S A G 12.1, h 36$, and 169 , are known as typical senescence markers, which are positively related to leaf senescence (Lee et al., 2001; Zhang et al., 2016; Zhou et al., 2013). Results of SL effects on leaf senescence are incomplete in the literature. Furthermore, there is limited information regarding the mechanisms of SL regulation of leaf senescence.

Leaf senescence is manifested by the decrease in chlorophyll caused by the decline in chlorophyll synthesis (Vajpayee et al., 2000) or the acceleration of chlorophyll degradation (Jespersen et al., 2016; Zhang et al., 2011). Chlorophyll degradation is controlled by multiple genes, including chlorophyllide a oxygenase $(N Y C 1)$, chlorophyll b reductase $(N O L)$, pheophytinase $(P P H)$, pheophorbide a oxygenase $(P A O)$, red chlorophyll catabolite reductase $(R C C R 1)$, and nonyellowing $1(S G R)$. Chlorophyll biosynthesis is regulated by multiple genes, including glutamyl-tRNA reductase (HEMA), glutamate-1semialdehyde 2,1- aminotransferase (GSA), 5-aminolevulinate dehydrogenase $(H E M B)$, uroporphyrinogen III decarboxylase (HEME), Mg-chelatase $(C H L H)$, and chlorophyll synthase (CHLG) (Yu et al., 2018; Zhu et al., 2015). Qiu et al. (2015) reported that ethylene insensitive3 (EIN3) was an important transcription factor in the ethylene-signaling pathway that promoted chlorophyll degradation via the direct increase of chlorophyll catabolic genes, such as NYC1 and PAO, in arabidopsis. However, whether leaf senescence affected by SL is due to inhibited chlorophyll synthesis genes and/or promoted chlorophyll degradation genes and senescence marker genes is not well-documented.

We hypothesized that SL may affect leaf senescence in perennial ryegrass (Lolium perenne) exposed to dark conditions by affecting chlorophyll biosynthesis and/or degradation independently or interactively with ethylene. Therefore, the objectives of this study were to examine the effects of SL on darkinduced leaf senescence and the chlorophyll metabolism of perennial ryegrass by an exogenous application of GR24 and to investigate the interactive effects of SL and ethylene in regulating leaf senescence in perennial grass exposed to dark conditions by exogenous treatment with an ethylene inhibitor, aminoethoxyvinyl glycine (AVG).

\section{Materials and Methods}

Plant material and treatments. Seeds of perennial ryegrass (cv. Pangaea) were planted in 30 plastic pots $(12 \times 12$ $\mathrm{cm}$ ) filled with potting mix including sphagnum peatmoss and humus (ProMix; Premier Horticulture, Quakertown, PA) on 10 Apr. 2018. Plants were maintained in growth chambers (Environmental Growth Chambers, Chagrin Falls, OH) controlled at $400 \mu \mathrm{mol} \cdot \mathrm{m}^{-2} \cdot \mathrm{s}^{-1}$ photosynthetically active radiation at temperatures of $22 / 18{ }^{\circ} \mathrm{C}$ (day/ night) with $60 \%$ relative humidity for a 14 -h photoperiod. All plants were well-watered and fertilized weekly with half-strength Hoagland's nutrient solution (Hoagland and Arnon, 1950).
To examine the effective concentrations of SL affecting dark-induced leaf senescence in perennial ryegrass, 4-week-old seedlings were sprayed with either distilled water (control) or solution containing $0.01,0.1,1,5$, or $10 \mu \mathrm{M}$ rac-GR24 (dissolved in acetone and diluted with water) before the exposure of plants to darkness. At each application, $40 \mathrm{~mL}$ of solution was sprayed onto leaves in each pot. The experiment was arranged as a completely randomized design with three replicates (three pots) for each treatment. Five pots of plants treated with five concentrations of GR24 were randomly placed in each of three growth chambers. The pots were relocated across and within the growth chamber every $2 \mathrm{~d}$ to avoid environmental variations in different chambers

To induce leaf senescence, plants treated with or without GR24 of different concentrations were exposed to dark conditions in growth chambers with lights off for $8 \mathrm{~d}$. To determine whether ethylene was involved in SL effects on dark-induced leaf senescence in perennial ryegrass, 4-week-old plants were treated with distilled water (control), $10 \mu \mathrm{M}$ GR24 (concentration was optimized by a pre-experiment), $5 \mu \mathrm{M}$ AVG (dissolved in water), or both GR24 and AVG on 12 May 2018, before plants were exposed to darkness. The experiment was arranged as a completely randomized design with three replicates (three pots of plants with multiple plants in each pot) for each treatment. Four pots of plants treated with water, GR24, AVG, or both GR24 and AVG were randomly placed in each of three growth chambers. The pots were relocated across and within the growth chamber every $2 \mathrm{~d}$ to avoid environmental variations in different chambers.

Measurements of Physiological Parameters. Fully expanded leaves were sampled on 20 May 2018, to measure the physiological parameters. Leaf chlorophyll content, photochemical efficiency (Fv/Fm), and electrolyte leakage (EL) were determined as physiological index measurements. Chlorophyll content was measured using leaves soaked in dimethyl sulfoxide (DMSO) in darkness for $72 \mathrm{~h}$ to extract chlorophyll. Then, a spectrophotometer (721G; Shanghai Jingke Instrument Plant, Shanghai, China) was used to measure the absorbance of extracts at 663 and $645 \mathrm{~nm}$. Chlorophyll content was calculated according to the equations published previously (Barnes et al., 1992). Fv/Fm was measured using a fluorescence meter (PAM2500; Walz, Effeltrich, Germany) as described by Oxborough and Baker (1997). To measure EL, $0.2 \mathrm{~g}$ of leaves were sampled. The leaves were washed three times and then immersed in deionized water $(35 \mathrm{~mL})$. After shaking for $24 \mathrm{~h}$, initial conductivity $(\mathrm{Ci})$ was determined with a conductivity meter (DDS-307A; Shanghai Jingke Instrument Plant). The maximum conductivity (Cmax) was measured after the leaves were boiled for $20 \mathrm{~min}$. The EL was determined as $100 \times \mathrm{Ci}$ / Cmax (Murray et al., 1989).

Measurement of ethylene Concentration. To measure ethylene production in leaves exposed to GR24 and AVG treatments, the blades of fully expanded leaves of perennial ryegrass were excised from whole plants that were treated with distilled water (control), $10 \mu \mathrm{M}$ GR24, $5 \mu \mathrm{M}$ AVG, or both GR24 and AVG and exposed to darkness for $8 \mathrm{~d}$. Leaf samples were wrapped in paper towels moistened with distilled water, GR24, AVG, or both GR24 and AVG; then, they were placed in an airtight $20-\mathrm{mL}$ glass vial for $2 \mathrm{~h}$ for the release of ethylene from leaves. A $100-\mu \mathrm{L}$ gas sample was collected from each vial using a syringe and injected in a gas chromatograph (GC) (6890N; Agilent Technologies, Santa Clara, CA) to analyze the 
ethylene concentration following the procedure described by Zaidi et al. (2016). The system consisted of a GC column, preconcentrator (PC), and $\mathrm{SnO}_{2}$ detector, with $\mathrm{PC}$ heated to $250^{\circ} \mathrm{C}$ for ethylene release.

Gene EXPRESSion ANALYSIS. The transcript levels of genes related to senescence, chlorophyll, ethylene, and SL in leaves were analyzed by a quantitative reverse-transcriptase polymerase chain reaction (qRT-PCR). Total RNA was extracted from leaf tissue with E.Z.N.A. Plant RNA Kit (Omega Bio-tek, Norcross, GA), and contaminating genomic DNA was removed with TURBO DNA-free reagent (Life Technologies, Grand Island, NY). Next, $2 \mu \mathrm{g}$ of total RNA was reverse-transcribed to cDNA using the high-capacity cDNA reverse-transcription kit (Life Technologies). During the gene expression analysis, a real-time PCR system (CFX CONNCT; Bio-Rad, Hercules, CA) was used and the PCR reaction was performed with a power SYBR Green PCR Master mix (Applied Biosystems, Foster City, CA). Primers used in this study are listed in Table 1 , and the data were standardized using eIF4A (Huang et al., 2014). The relative expression level between genes of interest and the reference gene was calculated using the $\Delta \Delta \mathrm{Ct}$ method. All reactions were performed with two technical and three biological replicates.

Statistical analysis. Effects of GR24 treatment and AVG treatment on all parameters were analyzed by an analysis of variance with SPSS software (version 20.0; IBM, Armonk, NY). Differences between mean values for each parameter were determined by a least significance test with $P=0.05$.

\section{Results}

EFFECTS OF GR24 AND ETHYLENE ON LEAF SENESCENCE IN PERENNIAL RYEGRASS EXPOSED TO DARKNESS. GR24 treatments lead to significant decreases in leaf chlorophyll content and photochemical efficiency (Fig. 1A and B) and increases in EL (Fig. 1C) in perennial ryegrass exposed to darkness. The effects

Table 1. Gene name and forward and reverse primer sequences used during the quantitative reversetranscriptase polymerase chain reaction analysis of gene expression in perennial ryegrass.

\begin{tabular}{|c|c|c|}
\hline Target gene & Forward primer $\left(5^{\prime}-3^{\prime}\right)$ & Reverse primer $\left(5^{\prime}-3^{\prime}\right)$ \\
\hline$\overline{L p S A G 12.1}$ & ACTGCGACACGACAGACAAC & TGTACTCGAAGGCATTGTCC \\
\hline Lph36 & GACCGCCCTTTGAACATAGT & CTTCAATCACGTCAGGATGG \\
\hline Lpl69 & TCATGGACAACATAGCAGCA & TACTTTCACGCCGTCGATAC \\
\hline $\mathrm{LpNYCl}$ & GATCGTCTCCCAGAAGTGCT & GCCAGTCTCCTGCTTGAAC \\
\hline LpNOL & GCTGGCAAAGAAGTTTCTCA & ATGCTGCTCTCCAAATTCCT \\
\hline$L p P P H$ & ACCCAGGTGATTCAGGAAAG & CCTGACCTCACCAACCTTCT \\
\hline$\angle p P A O$ & TCAAGGCCAAGAGAAGGTCT & TGTGTGGGTGTGAATGTGAG \\
\hline$L p R C C R l$ & ATGGTGCAATCGACATCACT & GAGCAGGTCCAGAATGACAA \\
\hline$L p S G R$ & GAGGAGGCGAACTCGAAG & GGTTGTACCACCCTTGCAG \\
\hline LpHEMA & TGCTACACGCCACCTGTTTGAG & TCCACTGTTTTGCCCGTTTCTG \\
\hline$L p G S A 1$ & GATGCCAAGAAGAGCGATAC & GTGAGCCAAGCTGGTAAAG \\
\hline LpHEMB & GTTCCAGGAGACCAGCATTACC & CTGAAGCACCCAGGCATAGC \\
\hline LpHEME & CGATATCTCGGAATCAAAGGG & CGACGAAGTGGAGCTTATC \\
\hline LpCHLH & CCAGACAAGGGCAATGTT & CGATGAGTTCTTCAGGTGTG \\
\hline$L p C H L G$ & GCCTTACCGTCCTATTCCTTCA & CCAACCCAAGACCTCCTAACAA \\
\hline $\operatorname{LpD17}$ & CGGCGATGCTCTGTCAAG & CCACGCTATCAAACGGAAA \\
\hline$L p D 3$ & AGTCTGCGTCCAAGAAATG & AATGAGCGGGTTGAGTTG \\
\hline LpD14 & CCTCGGTAGTCTGTCTCAT & CGCCAACCATCTTCTTCTT \\
\hline LpACO & GATTGCCCAGTCAACTACTAC & GCTGTTTGCAGCCAATTC \\
\hline LpEIN4 & GGCTTATTGTCCAGTCAAGAG & GAGGCCTTCTGGATCTGATA \\
\hline LpelF4A & AACTCAACTTGAAGTGTTGGAGTG & AGATCTGGTCCTGGAAAGAATATG \\
\hline
\end{tabular}

were more pronounced with increasing GR24 concentrations (Fig. 1). Leaves treated with GR24 appeared yellower compared with the untreated control (Fig. 2). Treatment with AVG alone and with AVG combined with GR24 maintained greener leaves compared with the GR24 treatment (Fig. 2). The AVG application ameliorated the senescence traits caused by GR24, causing $15.9 \%$ and $36.0 \%$ increases in chlorophyll content and photochemical efficiency, respectively (Fig. 2A and B), and a $4.1 \%$ decrease in EL compared with the GR24 treatment (Fig. 2C).

EFFECTS OF GR24 AND AVG ON ETHYLENE PRODUCTION OF LEAVES EXPOSED TO DARKNESS. To determine whether SLaccelerated leaf senescence is associated with ethylene production, ethylene evolution rates of leaves treated with GR24 or AVG were examined. As shown in Fig. 3, AVG inhibited ethylene production, causing a $78.9 \%$ decrease in the ethylene evolution rate. However, GR24 promoted ethylene production, with a $320 \%$ increase compared to the untreated control. The ethylene production rate was also significantly greater with the combined AVG and GR24 treatment compared with the untreated control, but it was significantly lower than that of plants treated with GR24 alone.

EXPRESSION OF GENES RELATED TO LEAF SENESCENCE. The transcript changes of three genes related to leaf senescence (LpSAG12.1, Lph36, and Lpl69) were compared among plants treated with or without GR24 or AVG. The relative expressions of all three genes were significantly increased in plants treated with GR24 alone, with a $201.3 \%$ increase for $L p S A G 12.1$, $369.0 \%$ increase for $L p h 36$, and $210.3 \%$ increase for Lpl69 (Fig. 4A-C). The AVG treatment caused downregulation of LpSAG12.1 but had no significant effects on Lph36, and Lpl69. The expression levels were significantly lower in plants treated with both AVG and GR24 compared with those treated with GR24 alone; levels were 87.2\% lower for $\operatorname{LPSAG12.1,25.5\% }$ lower for Lph36, and 12.3\% lower for Lpl69 (Fig. 4A-C).

EXPRESSION OF CHLOROPHYLL-RELATED GENES. To investigate whether GR24 or AVG may affect chlorophyll degradation and/or suppress chlorophyll biosynthesis, the different expression levels of six chlorophyll degradation-associated genes (LPNYC1, LpNOL, LpPPH, LPPAO, LpRCCR1, and LpSGR) and six chlorophyll biosynthesis-associated genes (LpHEMA, LpGSA, $L p H E M B, L p H E M E, L p C H L H$, and $L p C H L G$ ) were compared between plants treated with or without GR24, AVG, or a combination of GR24 and AVG. The relative expression levels of four chlorophyll degradation-associated genes ( $L p N O L$, $L p P P H, L p P A O$, and $L p R C C R 1)$ were increased with GR24 treatment (Fig. 5A), and the relative expression of all six chlorophyll degradation-associated genes were decreased with AVG treatment (Fig. 5A). Expression levels were significantly lower in plants treated with both AVG and GR24 compared with those treated with GR24 alone; 

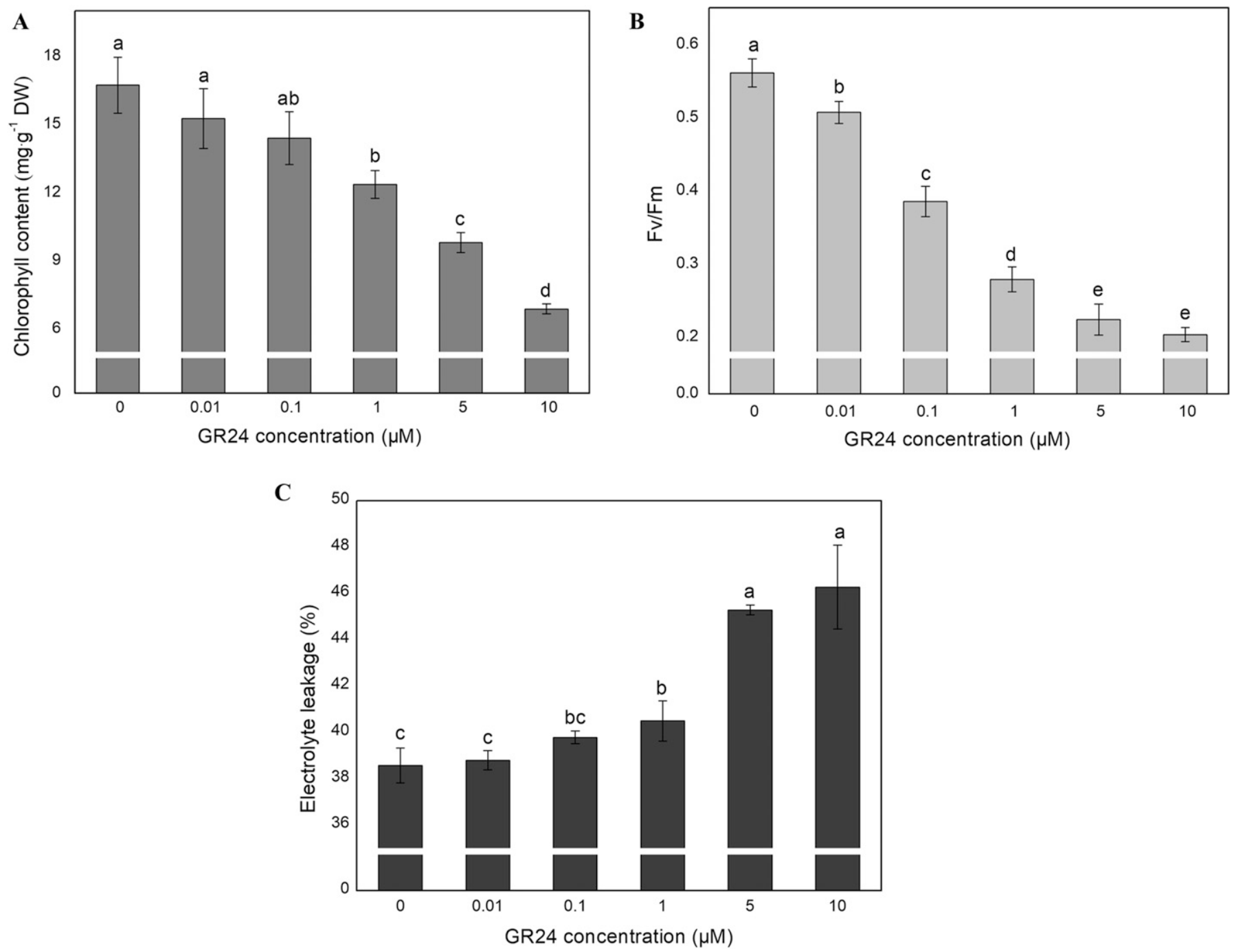

Fig. 1. Effects of $0,0.01,0.1,1,5$, and $10 \mu \mathrm{M}$ of a synthetic strigolactone analogue (GR24) on physiological parameters at day 8 after dark treatment in leaves of perennial ryegrass: (A) chlorophyll content, (B) photochemical efficiency $(\mathrm{Fv} / \mathrm{Fm})$, and $(\mathbf{C})$ electrolyte leakage. Bars represent the sE for three biological replicates. The lettering indicates significant differences when treatments were marked with different letters according to the Duncan multiple range test $(P \leq$ $0.05)$.

levels were $61.5 \%$ lower for $L p N Y C 1,34.2 \%$ lower for $L p N O L$, $34.6 \%$ lower for $L p P P H, 39.4 \%$ lower for $L p P A O, 29.1 \%$ lower for $L p R C C R 1$, and $61.3 \%$ lower for $L p S G R$ (Fig. $5 \mathrm{~A}$ ).

The relative expression of four chlorophyll biosynthesisassociated genes decreased with GR24 treatment compared with the untreated control, with a $42.5 \%$ decrease for $L p H E M A$, $18.9 \%$ decrease for $L p G S A, 34.7 \%$ decrease for $\angle p H E M B$, and $32.9 \%$ for $L p C H L H$ (Fig. 5B). AVG treatment significantly enhanced the relative expression of all six chlorophyll biosynthesisassociated genes. The expression levels of four chlorophyll biosynthesis-associated genes did not differ significantly between GR24 treatment and the combined AVG and GR24 treatment (Fig. 5B).

EXPRESSION OF SL-RELATED GENES AND ETHYLENE-RELATED GENES. To understand changes in SL-related genes and ethylene-related genes in response to GR24 or AVG, the expression levels of SL biosynthesis-associated gene $L p D 17$, SL signalingassociated genes $L p D 3$ and $L p D 14$, ethylene biosynthesisassociated gene $L P A C O$, and ethylene signaling-associated genes LPEIN4 and LpERS1 were analyzed. The relative ex- pression levels of $L p D 17, L p D 3$, and $L p D 14$ increased with GR24 treatment $(133 \%, 244 \%$, and $214 \%$ increase, respectively), but they decreased significantly with AVG treatment $(39.7 \%, 30.2 \%$, and $56.4 \%$, respectively) (Fig. 6A). The relative expression of ethylene-related genes increased with GR24 treatment, with an $81.4 \%$ increase for $L p A C O, 27.2 \%$ increase for LpEIN4, and 35.9\% increase for LpERS1 (Fig. 6B). AVG treatment suppressed the relative expression compared with the untreated control, with a $48.5 \%$ decrease for $L p A C O, 26.4 \%$ decrease for LpEIN4, and 59.5\% decrease for LpERSI (Fig. $6 \mathrm{~B})$. Plants treated with the combined AVG and GR24 treatment had significantly lower relative expression levels that those treated with GR24 alone, with $71.3 \%$ lower levels for LpEIN4 and 66.0\% lower levels for LpERSI (Fig. 6B).

\section{Discussion}

The physiological analysis showed that the exogenous GR24 application effectively accelerated leaf senescence in perennial 

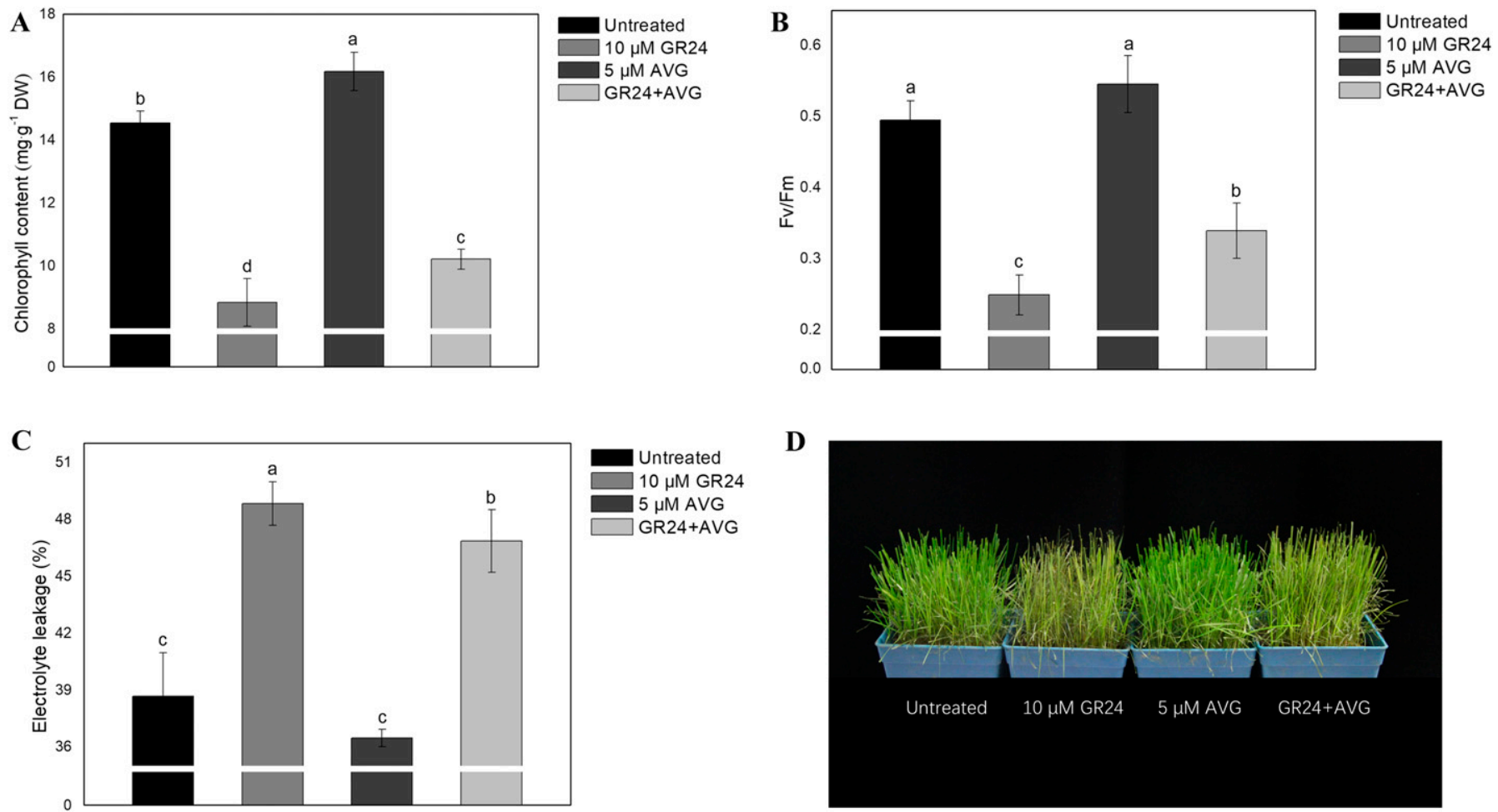

Fig. 2. Effects of a synthetic strigolactone analogue (GR24), aminoethoxyvinyl glycine (AVG), which is an ethylene biosynthesis inhibitor, and a combination of both on physiological parameters at day 8 after dark treatment in leaves of perennial ryegrass: (A) chlorophyll content, $(\mathbf{B})$ photochemical efficiency $(\mathrm{Fv} / \mathrm{Fm}),(\mathbf{C})$ electrolyte leakage, and (D) phenotype traits. Bars represent the SE for three biological replicates. The lettering indicates significant differences when treatments were marked with different letters according to the Duncan multiple range test $(P \leq 0.05)$.

ryegrass incubated in the dark, as characterized by the lower chlorophyll content, lower photochemical efficiency, and higher EL. The trend in expressions of senescence-related genes LpSAG12.1, Lph36, and Lpl69 also support this conclusion.

Ethylene acts as a positive regulator of leaf senescence (Jibran et al., 2013), and ethylene production has been considered an important factor contributing to senescence of leaves (Yang and Hoffman, 1984). SL may interact with ethylene, affecting leaf senescence (Kapulnik et al., 2011; Li et al., 2018; Ueda and Kusaba, 2015). In our studies, ethylene production rates increased significantly with the GR24 treatment, whereas the combined AVG and GR24 treatment decreased the production compared with the GR24 treatment. Ethylene biosynthesis-associated gene $L P A C O$ and ethylene signaling-associated genes LPEIN4 and LPERSI showed a similar trend (Fig. 6B). These data support the notion that SL could regulate dark-induced leaf senescence by promoting ethylene synthesis and signaling in perennial ryegrass. Kapulnik et al. (2011) suggested that SL promotes root hair elongation by enhancing ethylene produc$0.05)$.

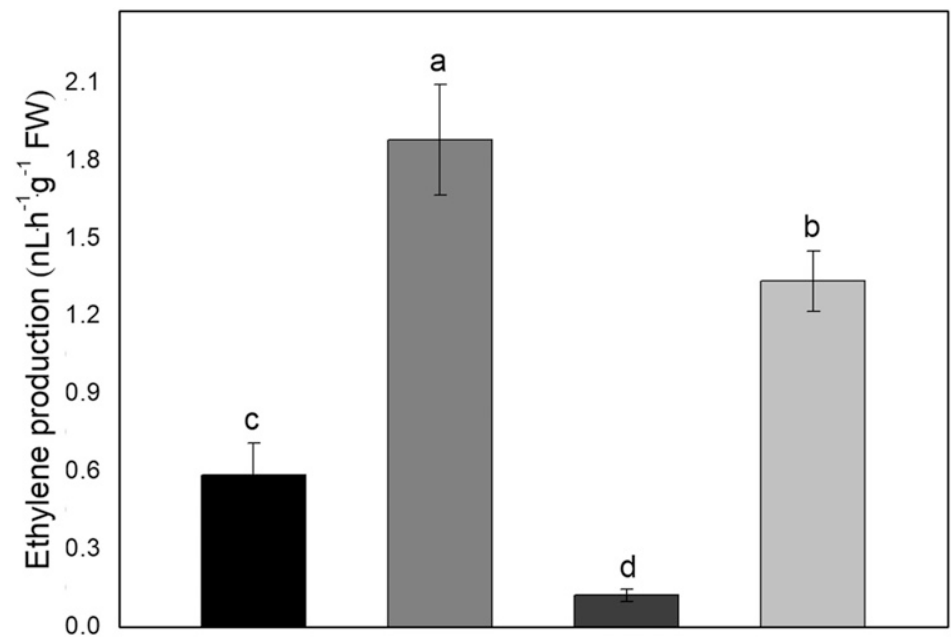

Untreated $10 \mu \mathrm{M}$ GR24 $5 \mu \mathrm{M}$ AVG $A V G+G R 24$

Fig. 3. Effects of a synthetic strigolactone analogue (GR24), aminoethoxyvinyl glycine (AVG), which is an ethylene biosynthesis inhibitor, and a combination of both on ethylene production of perennial ryegrass leaves at day 8 after dark treatment. Bars represent the se for three biological replicates. The lettering indicates significant differences when treatments were marked with different letters according to the Duncan multiple range test $(P \leq$

tion. Additionally, Sugimoto et al. (2003) reported that SL causes upregulated production of ethylene by inducing mRNA expression of ethylene biosynthesis-associated genes during germination of Striga hermonthica. Another study reported that the addition of AVG suppressed germination induced by SL in S. hermonthica (Logan and Stewart, 1991), which is consistent 

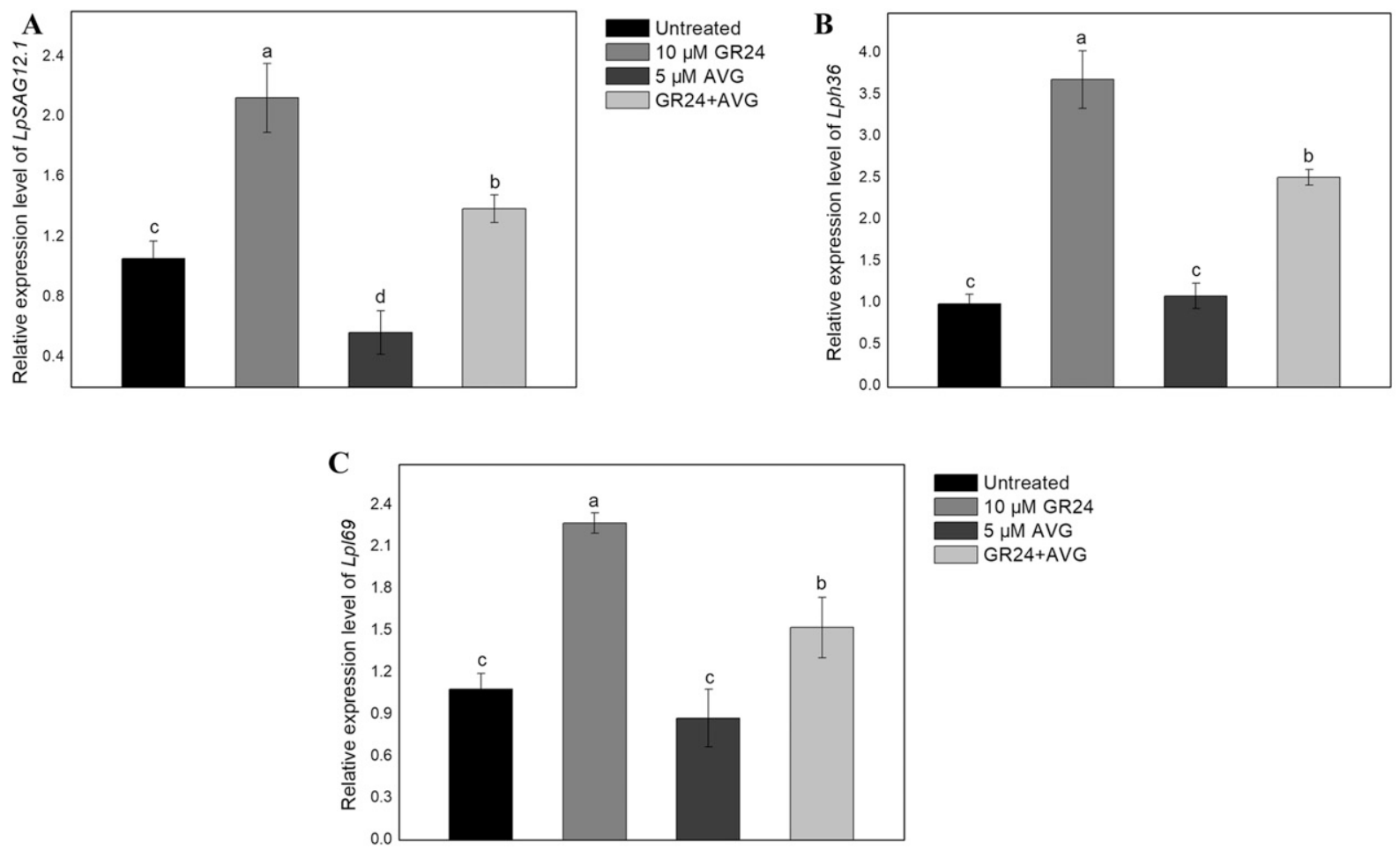

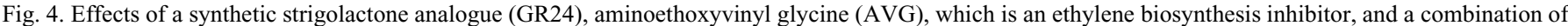
both on the relative expression of genes related to senescence including (A) LpSAG12.1, (B) Lph36, and (C) Lpl69 of perennial ryegrass at day 8 after dark treatment. Bars represent the sE for three biological replicates. The lettering indicates significant differences when treatments were marked with different letters according to the Duncan multiple range test $(P \leq 0.05)$.
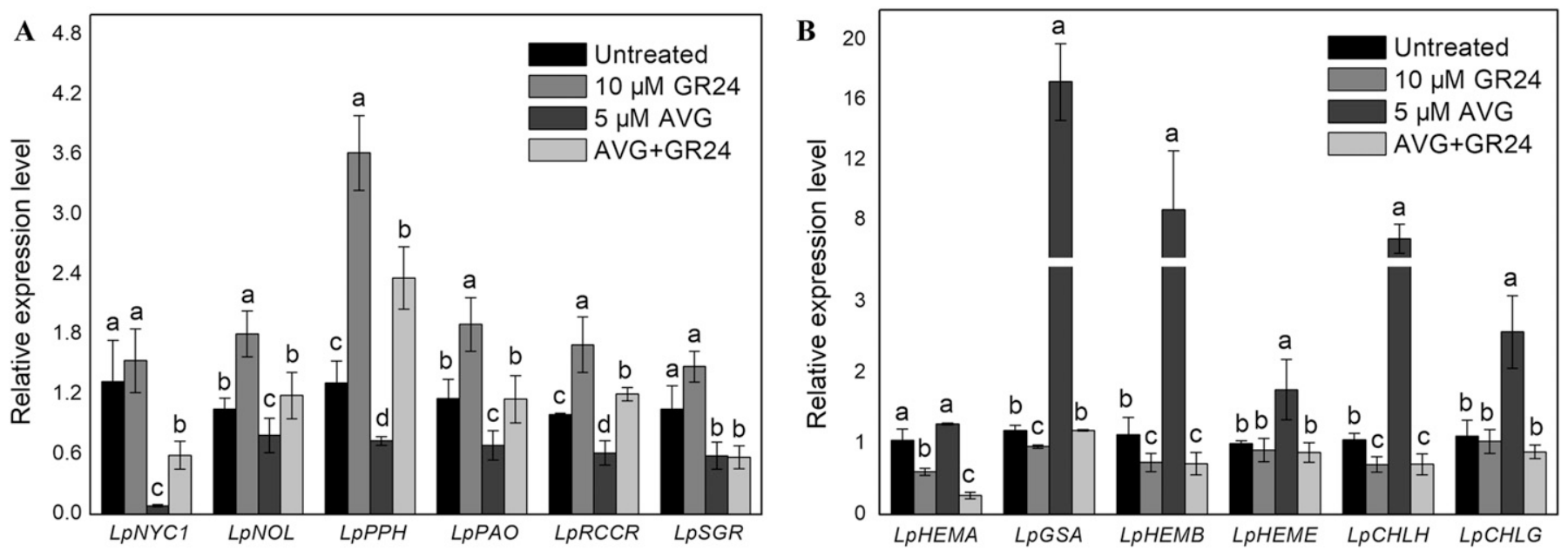

Fig. 5. Effects of a synthetic strigolactone analogue (GR24), aminoethoxyvinyl glycine (AVG), which is an ethylene biosynthesis inhibitor), and a combination of both on the relative expression of (A) chlorophyll degradation-associated genes ( $L p N Y C 1, L p N O L, L p P P H, L p P A O, L p R C C R 1$, and $L p S G R)$ and (B) chlorophyll biosynthesis-associated genes ( $L p H E M A$, LpGSA, LpHEMB, LpHEME, LpCHLH, and $L p C H L G$ ) of perennial ryegrass at day 8 after dark treatment. Bars represent the SE for three biological replicates. The lettering indicates significant differences when treatments were marked with different letters according to the Duncan multiple range test $(P \leq 0.05)$.

with the leaf senescence results found during our study. These studies suggested that the synergistic effects of SL and ethylene may be consistent during the development of plants.

A transcript analysis of SL biosynthesis and signalingassociated genes demonstrated that AVG application downregulated the expression of SL biosynthesis-associated gene LpD17, which is consistent with a previous study of arabidopsis
(Kapulnik et al., 2011; Li et al., 2018; Ueda and Kusaba, 2015). This indicated that ethylene also promoted SL biosynthesis in a monocotyledon plant such as perennial ryegrass. In addition, the expression of SL signaling-associated genes, including $L p D 3$ and $L p D 14$, were also repressed with AVG application. Together, these results suggest that an SL-dependent pathway also contributes to ethylene-induced senescence. 

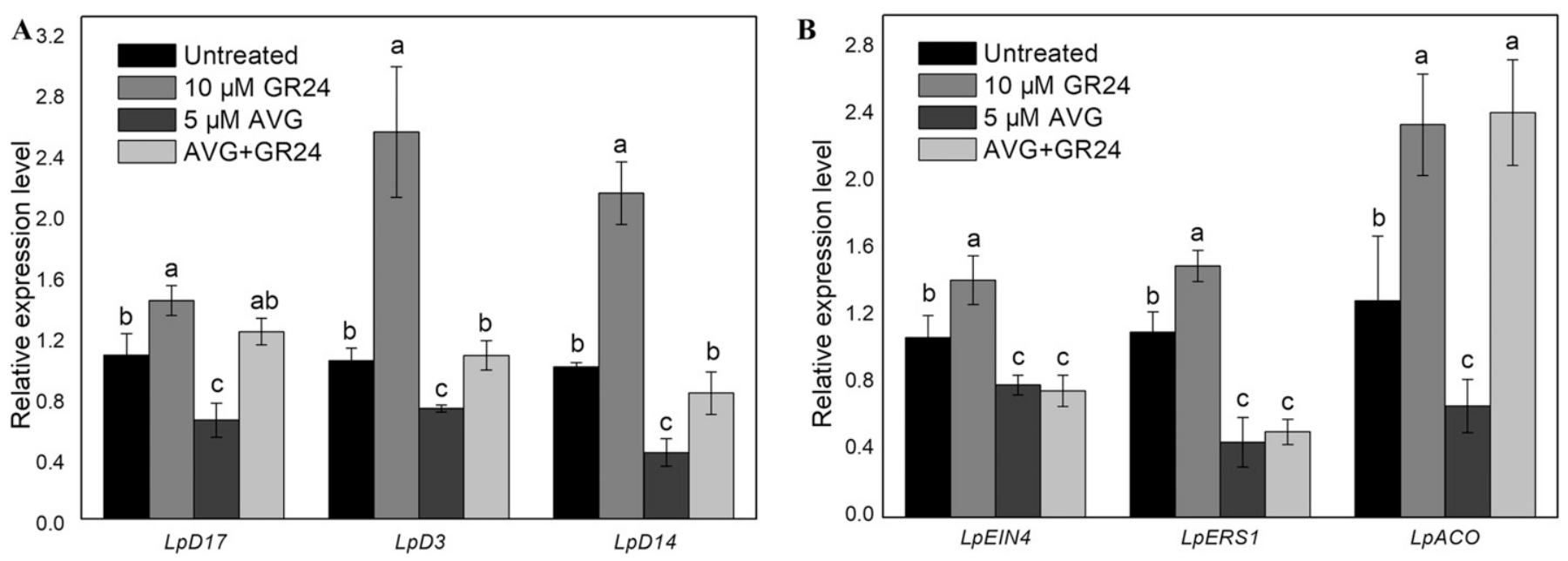

Fig. 6. (A) Effects of a synthetic strigolactone analogue (GR24), aminoethoxyvinyl glycine (AVG), which is an ethylene biosynthesis inhibitor), and a combination of both on the relative expression of SL biosynthesis-related genes $L p D 17$ and SL signaling-associated genes ( $L p D 3$ and $L p D 14$ ) of perennial ryegrass after dark treatment. (B) Effects of GR24, AVG, and a combination of both on the relative expression of ethylene biosynthesis-related genes $(L p A C O)$ and ethylene signaling-associated genes ( $L p E I N 4$ and $L p E R S 1$ ) of perennial ryegrass after dark treatment. Bars represent the sE for three biological replicates. The lettering indicates significant differences when treatments were marked with different letters according to the Duncan multiple range test $(P \leq 0.05)$.

In our study, GR24 treatment resulted in reduced expression of four chlorophyll biosynthesis-associated genes and upregulation of four chlorophyll degradation-associated genes in perennial ryegrass grown in the dark. Upregulation of chlorophyll degradation-associated genes caused by SL could be ameliorated by AVG treatment, but AVG had no obvious antagonistic effects on SL for chlorophyll biosynthesis-associated genes. These results indicated that SL may have an adverse effect on chlorophyll biosynthesis-associated genes and a positive effect on chlorophyll degradation-associated genes, thus leading to a lower chlorophyll content. However, the decrease could be relieved by AVG through suppressing SL upregulation of chlorophyll degradation-associated genes. The expression levels of $P A O, P P H$, and $R C C R$ genes associated with chlorophyll degradation were enhanced with ethylene treatment in chinese flowering cabbage [Brassica rapa var. parachinensis (Zhang et al., 2011)] and broccoli [Brassica oleracea var. italica (Buchert et al., 2011)]. Ethylene has been shown to create a several-fold increase in chlorophyllase activity that accelerates chlorophyll breakdown (Amir-Shapira et al., 1987; Purvis and Barmore, 1981; Shimokawa et al., 1978); however, chlorophyll synthesis was slightly inhibited by ethylene treatment (Alscher and Castelfranco, 1972). Based on all of these studies, we suggest that SL promotes leaf senescence through the acceleration of chlorophyll degradation in concert with ethylene. Moreover, SL is involved in an ethyleneindependent, senescence-promoting pathway through downregulating chlorophyll synthesis under dark conditions.

\section{Conclusions}

We found that exogenously applied GR24 promotes leaf senescence in the dark in perennial ryegrass, and that the intensity of action increased with the GR24 concentration. SLaccelerated leaf senescence was associated with the downregulation of four chlorophyll biosynthesis-associated genes and upregulation of four chlorophyll degradation-associated genes. AVG had functions counteractive to SL, thus suppress- ing leaf senescence by downregulating chlorophyll degradation genes and SL synthesis genes. Our results suggest that SL and ethylene interactively regulated leaf senescence, mainly by controlling chlorophyll degradation induced by darkness in perennial ryegrass. However, the underlying mechanisms of whether and how SL and ethylene directly interact with each other to control stress-induced leaf senescence deserve further investigation.

\section{Literature Cited}

Alscher, R.G. and P.A. Castelfranco. 1972. Stimulation by ethylene of chlorophyll biosynthesis in dark-grown cucumber cotyledons. Plant Physiol. 50:400-403.

Amir-Shapira, D., E.E. Goldschmidt, and A. Altman. 1987. Chlorophyll catabolism in senescing plant tissues: In vivo breakdown intermediates suggest different degradative pathways for citrus fruit and parsley leaves. Proc. Natl. Acad. Sci. USA 84:1901-1905.

Barnes, J.D., L. Balaguer, E. Manrique, S. Elvira, and A.W. Davison. 1992. A reappraisal of the use of DMSO for the extraction and determination of chlorophylls $\mathrm{a}$ and $\mathrm{b}$ in lichens and higher plants. Environ. Exp. Bot. 32:85-100.

Beveridge, C.A., I.C. Murfet, L. Kerhoas, B. Sotta, E. Miginiac, and C. Rameau. 1997. The shoot controls zeatin riboside export from pea roots. Evidence from the branching mutant rms4. Plant J. 11:339-345.

Buchert, A.M., P.M. Civello, and G.A. Martinez. 2011. Chlorophyllase versus pheophytinase as candidates for chlorophyll dephytilation during senescence of broccoli. J. Plant Physiol. 168:337-343.

Cook, C., L.P. Whichard, B. Turner, M.E. Wall, and G.H. Egley. 1966. Germination of witchweed (Striga lutea Lour.): Isolation and properties of a potent stimulant. Science 154:1189-1190.

Ha, C.V., M.A. Leyva-González, Y. Osakabe, U.T. Tran, R. Nishiyama, Y. Watanabe, M. Tanaka, M. Seki, S. Yamaguchi, N.V. Dong, K. Yamaguchi-Shinozaki, K. Shinozaki, L. Herrera-Estrella, and L.-S.P. Tran. 2014. Positive regulatory role of strigolactone in plant responses to drought and salt stress. Proc. Natl. Acad. Sci. USA 111:851.

Hoagland, D.R. and D.I. Arnon. 1950. The water-culture method for growing plants without soil. California Agr. Exp. Sta. Circ. 347:357-359. $\mathrm{Hu}$, Q., S. Zhang, and B. Huang. 2018. Strigolactones and interaction with auxin regulating root elongation in tall fescue under different temperature regimes. Plant Sci. 271:34-39. 
Huang, L., H. Yan, X. Jiang, G. Yin, X. Zhang, Q. Xiao, Y. Zhang, Y. Yan, X. Ma, and Y. Peng. 2014. Identification of candidate reference genes in perennial ryegrass for quantitative RT-PCR under various abiotic stress conditions. PLoS One 9:e93724.

Jespersen, D., J. Zhang, and B. Huang. 2016. Chlorophyll loss associated with heat-induced senescence in bentgrass. Plant Sci. 249:1-12.

Jibran, R., D.A. Hunter, and P.P. Dijkwel. 2013. Hormonal regulation of leaf senescence through integration of developmental and stress signals. Plant Mol. Biol. 82:547-561.

Kapulnik, Y., J. Hershenhorn, E. Mayzlish-Gati, S. Wininger, Y. Kaplan, N. Resnick, and H. Koltai. 2011. Strigolactones interact with ethylene and auxin in regulating root-hair elongation in Arabidopsis. J. Expt. Bot. 62:2915-2924.

Kohlen, W., T. Charnikhova, M. Lammers, T. Pollina, P. Tóth, I. Haider, M.J. Pozo, R.A. de Maagd, C. Ruyter-Spira, H.J. Bouwmeester, and J.A. Lopez-Raez. 2012. The tomato carotenoid cleavage dioxygenase8 (SlCCD8) regulates rhizosphere signaling, plant architecture and affects reproductive development through strigolactone biosynthesis. New Phytol. 196:535-547.

Lee, R.H., C.H. Wang, L.T. Huang, and S.C.G. Chen. 2001. Leaf senescence in rice plants: Cloning and characterization of senescence up-regulated genes. J. Expt. Bot. 52:1117-1121.

Li, W., R. Nishiyamaa, Y. Watanabea, C.V. Ha, M. Kojima, P. An, L. Tian, C.J. Tian, H. Sakakibara, and L.S.P. Tran. 2018. Effects of overproduced ethylene on the contents of other phytohormones and expression of their key biosynthetic genes. Plant Physiol. Biochem. 128:170-177.

Liu, J., M. Novero, T. Charnikhova, A. Ferrandino, A. Schubert, C. Ruyter-Spira, P. Bonfante, C. Lovisolo, H.J. Bouwmeester, and F. Cardinale. 2013. Carotenoid cleavage dioxygenase 7 modulates plant growth, reproduction, senescence, and determinate nodulation in the model legume Lotus japonicus. J. Expt. Bot. 64:1967-1981.

Logan, D.C. and G.R. Stewart. 1991. Role of ethylene in the germination of the hemiparasite Striga hermonthica. Plant Physiol. 97:1435-1438.

Murray, M.B., J.N. Cape, and D. Fowler. 1989. Quantification of frost damage in plant tissues by rates of electrolyte leakage. New Phytol. 113:307-311.

Oxborough, K. and N.R. Baker. 1997. Resolving chlorophyll a fluorescence images of photosynthetic efficiency into photochemical and non-photochemical components - Calculation of qP and Fv-/Fm-; without measuring Fo. Photosynth. Res. 54:135-142.

Purvis, A.C. and C.R. Barmore. 1981. Involvement of ethylene in chlorophyll degradation in peel of citrus fruits. Plant Physiol. 68:854-856.

Qiu, K., Z. Li, Z. Yang, J. Chen, S. Wu, X. Zhu, S. Gao, J. Gao, G. Ren, B. Kuai, and X. Zhou. 2015. EIN3 and ORE1 Accelerate degreening during ethylene-mediated leaf senescence by directly activating chlorophyll catabolic genes in Arabidopsis. PLoS Genet. 11:e1005399.

Shimokawa, K., S. Shimada, and K. Yaeo. 1978. Ethylene-enhanced chlorophyllase activity during degreening of Citrus unshiu Marc. Scientia Hort. 8:129-135.

Snowden, K.C., A.J. Simkin, B.J. Janssen, K.R. Templeton, H.M. Loucas, J.L. Simons, S. Karunairetnam, A.P. Gleave, D.G. Clark, and H.J. Klee. 2005. The Decreased apical dominance1/Petunia hybrida carotenoid cleavage dioxygenase 8 gene affects branch production and plays a role in leaf senescence, root growth, and flower development. Plant Cell 17:746-759.

Snowden, K.C., R.S.M. Drummond, S.E. Ledger, J.M. Cooney, C. Hamiaux, B.J. Janssen, and R.D. Newcomb. 2012. DAD2 is an alpha/ beta hydrolase likely to be involved in the perception of the plant branching hormone, strigolactone. Curr. Biol. 22:2032-2036.

Sugimoto, Y., A.M. Ali, S. Yabuta, H. Kinoshita, S. Inanaga, and A. Itai. 2003. Germination strategy of Striga hermonthica involves regulation of ethylene biosynthesis. Physiol. Plant. 119:137-145.
Sun, H., J. Tao, S. Liu, S. Huang, S. Chen, X. Xie, K. Yoneyama, Y. Zhang, and G. Xu. 2014. Strigolactones are involved in phosphateand nitrate-deficiency-induced root development and auxin transport in rice. J. Expt. Bot. 65:6735-6746.

Toh, S., Y. Kamiya, N. Kawakami, E. Nambara, P. McCourt, and Y. Tsuchiya. 2012. Thermoinhibition uncovers a role for strigolactones in arabidopsis seed germination. Plant Cell Physiol. 53:107-117.

Ueda, H. and M. Kusaba. 2015. Strigolactone regulates leaf senescence in concert with ethylene in arabidopsis. Plant Physiol. 169:138-147. Umehara, M., A. Hanada, S. Yoshida, K. Akiyama, T. Arite, N. Takeda-Kamiya, H. Magome, Y. Kamiya, K. Shirasu, K. Yoneyama, J. Kyozuka, and S. Yamaguchi. 2008. Inhibition of shoot branching by new terpenoid plant hormones. Nature 455:195.

Vajpayee, P., R.D. Tripathi, U.N. Rai, M.B. Ali, and S.N. Singh. 2000. Chromium (VI) accumulation reduces chlorophyll biosynthesis, nitrate reductase activity and protein content in Nymphaea alba L. Chemosphere 41:1075-1082.

Vogel, J.T., M.H. Walter, P. Giavalisco, A. Lytovchenko, W. Kohlen, T. Charnikhova, A.J. Simkin, C. Goulet, D. Strack, H.J. Bouwmeester, A.R. Fernie, and H.J. Klee. 2010. SlCCD7 controls strigolactone biosynthesis, shoot branching and mycorrhiza-induced apocarotenoid formation in tomato. Plant J. 61:300-311.

Wingler, A., A. von Schaewen, R.C. Leegood, P.J. Lea, and W.P. Quick. 1998. Regulation of leaf senescence by cytokinin, sugars, and light: Effects on NADH-dependent hydroxypyruvate reductase. Plant Physiol. 116:329-335.

Woo, H.R., K.M. Chung, J.H. Park, S.A. Oh, T. Ahn, S.H. Hong, S.K. Jang, and H.G. Nam. 2001. ORE9, an F-box protein that regulates leaf senescence in Arabidopsis. Plant Cell 13:1779-1790.

Xie, X., K. Yoneyama, and K. Yoneyama. 2010. The strigolactone story. Annu. Rev. Phytopathol. 48:93-117.

Yamada, Y., S. Furusawa, S. Nagasaka, K. Shimomura, S. Yamaguchi, and M. Umehara. 2014. Strigolactone signaling regulates rice leaf senescence in response to a phosphate deficiency. Planta 240:399408.

Yan, H., H. Saika, M. Maekawa, I. Takamure, N. Tsutsumi, J. Kyozuka, and M. Nakazono. 2007. Rice tillering dwarf mutant dwarf3 has increased leaf longevity during darkness-induced senescence or hydrogen peroxide-induced cell death. Genes Genet. Syst. 82:361-366.

Yang, S.F. and N.E. Hoffman. 1984. Ethylene biosynthesis and its regulation in higher plants. Annu. Rev. Plant Physiol. 35:155-189.

Yu, Q., Y. Shen, Q. Wang, X. Wang, L. Fan, Y. Wang, S. Zhang, Z. Liu, and M. Zhang. 2018. Light deficiency and waterlogging affect chlorophyll metabolism and photosynthesis in Magnolia sinostellata. Trees 33:1-12.

Zaidi, N.A., M.W. Tahir, P.P. Vinayaka, F. Lucklum, M. Vellekoop, and W. Lang. 2016. Detection of ethylene using gas chromatographic system. Procedia Eng. 168:380-383.

Zhang, J., H. Li, B. Xu, J. Li, and B. Huang. 2016. Exogenous melatonin suppresses dark-induced leaf senescence by activating the superoxide dismutase-catalase antioxidant pathway and down-regulating chlorophyll degradation in excised leaves of perennial ryegrass (Lolium perenne L.). Front. Plant Sci. 7:1500.

Zhang, X., Z. Zhang, J. Li, L. Wu, J. Guo, L. Ouyang, Y. Xia, X. Huang, and X. Pang. 2011. Correlation of leaf senescence and gene expression/activities of chlorophyll degradation enzymes in harvested chinese flowering cabbage (Brassica rapa var. parachinensis). J. Plant Physiol. 168:2081-2087.

Zhou, Q., Q. Yu, Z. Wang, Y. Pan, W. Lv, L. Zhu, R. Chen, and G. He. 2013. Knockdown of GDCH gene reveals reactive oxygen speciesInduced leaf senescence in rice. Plant Cell Environ. 36:1476-1489.

Zhu, X., J. Chen, Z. Xie, J. Gao, G. Ren, S. Gao, X. Zhou, and B. Kuai. 2015. Jasmonic acid promotes degreening via MYC 2/3/4- and ANAC 019/055/072-mediated regulation of major chlorophyll catabolic genes. Plant J. 84:597-610. 